\title{
ON THE ALEXANDER POLYNOMIALS OF ALTERNATING TWO-COMPONENT LINKS
}

\author{
MARK E. KIDWELL \\ Department of Mathematics \\ Amherst College \\ Amherst, Massachusetts 01002 U.S.A.
}

(Received September 5, 1978)

ABSTRACT. Let $\mathrm{L}$ be an alternating two-component link with Alexander polynomial $\Delta(\mathrm{x}, \mathrm{y})$. Then the polynomials $(1-\mathrm{x}) \Delta(\mathrm{x}, \mathrm{y})$ and $(1-\mathrm{y}) \Delta(\mathrm{x}, \mathrm{y})$ are alternating. That is, $(1-y) \Delta(x, y)$ can be written as $\sum_{i, j} c_{i j} x^{i} y^{j}$ in such a way that $(-1)^{i+j} c_{i j} \geq 0$.

KEY WORDS AND PHRASES. Alternating link projection, Alexander matrix and polynomial, Adjacency matrix, rooted tree.

AMS (MOS) SUBJECT CLASSIFICATION (1970) CODES. Primary 55 A25.

\section{INTRODUCTION.}

This paper extends the graphical methods of Crowell [1] from the study of the reduced Alexander polynomial $\Delta(t)$ to the study of the unreduced Alexander polynomial $\Delta(x, y)$. This extension requires extra care in labelling the edges of our graph and in comparing its adjacency matrix to the Alexander matrix of the alternating link. 
Crowell also used his method to prove that for alternating links, $\operatorname{deg} \Delta(t)=2 h$, where $h$ is the genus. In a future paper, we hope to prove equalities relating the $\mathrm{x}$ - and $\mathrm{y}$-degrees of $\Delta(\mathrm{x}, \mathrm{y})$ to geometric properties of an alternating link.

The author would like to thank James Bailey and Joan Hutchinson for their helpful comments.

2. ALTERNATING LINK PROJECTIONS.

Let $P$ be an alternating, regular, planar projection of a two-component link $L=K_{1} U_{2}$, such as the projection of the link $6_{3}^{2}[4, p .416]$ in Fig. 1 . The components of $\mathrm{L}$ are oriented, and $\mathrm{P}$ inherits this link-orientation. The two thick arrows off the projection in Fig. I indicate link orientation. We call $\mathrm{K}_{1}$ the $\mathrm{x}$-component and $\mathrm{K}_{2}$ the $\mathrm{y}$-component.

The crossings of a projection are either positive, as shown in Fig. 2a), or negative, as shown in Fig. $2 \mathrm{~b}$ ), depending upon the orientations of the constituent segments. We also distinguish four types of crossings depending upon how the $\mathrm{x}$ - and $\mathrm{y}$-components enter in. These are:

$$
\begin{aligned}
& r_{x} \text {-crossings : } x \text {-component overcrosses } y \text {-component. } \\
& r_{y} \text {-crossings : y-component overcrosses } x \text {-component. } \\
& s_{x} \text {-crossings : } x \text {-component overcrosses itself. } \\
& s_{y} \text { - crossings : y-component overcrosses itself. }
\end{aligned}
$$

The capital letters $R_{x}, R_{y}, S_{x}$, and $S_{y}$ denote the number of crossings of each type. We follow Crowell's convention that if $R_{x}=R_{y}=0$ for a projection $P$, then $P$ is not alternating.

We shall need the following not-quite-obvious fact:

IEMMA 2.1. In an alternating link projection $P, R_{x}=R_{y}$. 
PROOF. Let $0_{x}$ be the union of the $r_{x}-$ and $s_{x}$-crossings and let $U_{x}$ be the union of the $\mathrm{r}_{\mathrm{y}}-$ and $\mathrm{s}_{\mathrm{x}}$-crossings. Let (i) be a crossing in $0_{x^{\circ}}$ The overcrossing $x$-component at (i) must terminate (go under) at an adjacent vertex $(j) \varepsilon U_{x}$, since the projection is alternating. Define $f((i))=(f)$. $f$ is a one-to-one correspondence between $O_{x}$ and $U_{x}$, so $R_{x}+S_{x}=R_{y}+S_{x}$ and $R_{x}=R_{y}$.

If a projection has d crossings labelled (1),(2),.., (d), we stipulate that (1) be an $r_{x}$-crossing, (2),.., (2R $\left.\mathrm{R}_{\mathrm{x}}\right)$ be $\mathbf{r}_{\mathrm{x}}-$ or $\mathrm{r}_{\mathrm{y}}$-crossings, and $\left(2 R_{x}+1\right), \ldots,(d)$ be $s_{x}-$ or $s_{y}$-crossings.

A Wirtinger presentation [2, p.86] for the link group $\Pi_{1}\left(S^{3}-L\right)$ has one generator for each overcrossing segment in a regular projection of the link. If the projection is alternating, we can label the segment which overcrosses at vertex (i) " $x_{i}$ " or " $y_{i}$ ", depending on whether it belongs to the $x-$ or y-component of the link. (Thus we define only one of the two symbols " $x_{i} "$, $\left.y_{i} ".\right)$ The relators $r_{i}, i=1, \ldots, d$, of the Wirtinger presentation are of the form

$$
r_{i}=x_{j} y_{i} x_{k}^{-1} y_{i}^{-1}
$$

for the crossing of Fig. 2a) and of the form

$$
r_{i}=x_{i} y_{j} x_{i}^{-1} y_{k}^{-1}
$$

for the crossing of Fig. 2b).

\section{ASSOCIATED GRAPH THEORY.}

We now regard the projection $P$ as a graph with the crossings of $P$ as vertices and the segments of $P$ joining vertices as edges. Thus each overcrossing segment in an alternating projection contributes two edges to the graph. We will use the word "vertex" when we are thinking of $P$ strictly as 
a graph, and the word "crossing" when we are thinking of $P$ as a link projection.

We next orient and label the edges of the graph of $P$. This alternating orientation differs from the link orientation. At each vertex (i) of $P$, we orient the two incident edges which belong to undercrossing segments at (i) toward (i). The edge which is on the left with respect to the overcrossing segment receives the label "l". The edge on the right receives the label "-x" if the overcrossing segment belongs to the $x$-component and "- $y$ " if the overcrossing segment belongs to the y-component. Fig. I shows the alternating orientation and labelling of $6_{3}^{2}$. We indicate the alternating orientation by arrows on the edges of $P$. We differ from Crowell [1, pp.260-61] only in using "-x" and "-y" where he uses simply "-t".

The adjacency matrix $B=\left(b_{i j}\right)$ of an oriented and labelled graph is the $d \times d$ matrix having for $(i, j)$ entry, $i \neq j$, the sum of the labels on the edges which start at $j$ and end at $i$. (If there are no such edges, then $\left.b_{i j}=0.\right)$ We also define $b_{i i}=-\sum_{j \neq i} b_{i j}$. Thus each row-sum of $B$ is zero.

THEOREM 3.1 (Matrix-tree theorem). Let $B^{\prime}$ be the matrix obtained from an adjacency matrix $B$ by crossing out the first row and column. Then $\operatorname{det} B^{\prime}=\sum_{T} \Pi(T)$, where the summation is over all maximal rooted trees $T$ with origin (1) in the graph [1, footnote 2, p. 262] and $\Pi(T)$ denotes the product of the labels on the edges of $T$.

PROOF. See [3, p.47]. Our matrix $B$ is the transpose of Crowell's (disregarding the difference in labelling). Crowell makes his column-sums zero while Moon [3] makes his row-sums zero. This discrepancy arises because Crowell is counting trees oriented away from the origin (as we are) while Moon is counting trees oriented toward the origin. 


\section{COMPARISON WITH THE ALEXANDER MATRIX.}

Our next task is to prove that the polynomial det $\mathrm{B}^{\prime}$ given in the last theorem is a link-type invariant.

The Alexander matrix A $[2, \mathrm{p} .100]$ of $\mathrm{L}$ with respect to the projection $\mathrm{P}$ and its Wirtinger presentation is the $d \times d$ matrix $\left(a_{i j}\right)=\left(\alpha \gamma\left(\frac{\partial r_{i}}{\partial x_{j}}\right)\right)$. (The $\operatorname{map} \gamma: F_{d}+\pi_{l}\left(S^{3}-L\right)$ displays the link group as a homomorphic image of a free group, and the Abelianizing map $\alpha$ sends all $x_{i} \rightarrow x$ and all $\left.y_{i}+y_{\cdot}\right)$ The rows and columns of $A$ correspond naturally to the vertices (1),..,(d) of $P$ since the relators and generators of the Wirtinger presentation so correspond. Define $\bar{A}$ to be the matrix obtained from $A$ by multiplying any row which corresponds to a negative crossing by -1 . From now on, we will call $\bar{A}$ the Alexander matrix.

LEMMA 4.1. Let $\bar{A}$ ' be the matrix obtained from $\bar{A}$ by deleting the first row and column. Then there is a polynomial $\Delta(x, y)$ such that, up to units $\pm x^{ \pm p} y^{ \pm q}$ in $\mathbb{Z}\left[x, x^{-1}, y, y^{-1}\right]$, det $\bar{A}^{\prime}=(1-x) \Delta(x, y)$.

PROOF. This is a special case of a theorem of Torres $[5$, p.61].

$\Delta(x, y)$ is defined to be the Alexander polynomial of the link. It is a link-type invariant [2, p.120]. If we had deleted a column of $\bar{A}$ corresponding to a segment from the $y$-component, we would have gotten $\operatorname{det} \bar{A}^{\prime}=(1-y) \Delta(x, y)$. THEORFM 4.2. For a given alternating link projection, the adjacency matrix $B$ and the Alexander matrix $\bar{A}$ are identical, except for the diagonal entries in

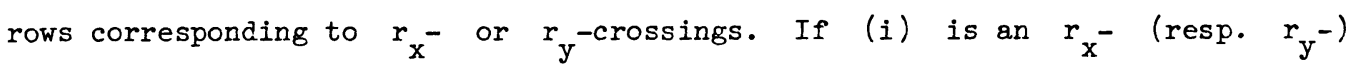
crossing, then $b_{i i}=x-1$ (resp. $y-1$ ) while $a_{i i}=y-1$ (resp. $x-1$ ).

PROOF. This is a routine application of the free differential calculus, and exactly follows [ $1,(2.10), p .261]$. Notice that $b_{i i}$ is defined to be minus the sum of the labels on all edges coming in to vertex ( $i$ ). This sum must be either $\mathrm{x}-1$ or $\mathrm{y}-1$. 
We give below the matrices $\bar{A}$ and $B$ for the alternating projection of the link $6_{3}^{2}$ shown in Fig. 1 .

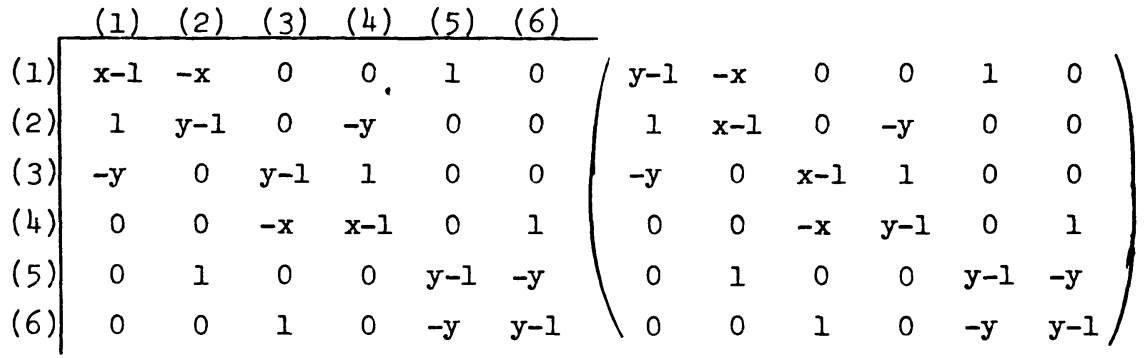

$$
\begin{aligned}
& \text { B } \bar{A}
\end{aligned}
$$

Recall that $\operatorname{det} B^{\prime}=\sum_{\sigma \varepsilon \Sigma}(-1)^{\circ} \prod_{i=2}^{d} b_{i 0(i)}$, where $\Sigma$ is the group of permutations of $\{2,3, \ldots, d\}$. Let $b_{0}=\prod_{i=2}^{d} b_{i 0(i)}$. Any permutation 0 can be written as a product of (algebraic) cycles $(i, o(i), o(o(i)), \ldots)(j, o(j)$, $o(o(j)), \ldots) \ldots$. A (geometric) cycle in a graph is a collection of oriented edges which forms a closed curve.

LEMMA 4.3. Let $b_{0}$ be a non-zero entry in det $B^{\prime}$. Then for every nontrivial cycle $(i, O(i), \ldots)$ in $o$, there is a cycle of edges in $P$ joining $\operatorname{vertex}(i)$ to vertex $o(i)$, etc.

PROOF. This is clear from the definition of the adfacency matrix.

In Fig. I, hashmarks indicate the (geometric) cycles corresponding to $0=(34)(56)$. For this example, $b_{0}=(y-1)(1)(-x)(-y)(-y)$.

LEMMA 4.4. Suppose there is an edge of the graph of $P$ joining vertex (i) to vertex $o(i)$. Then if $(i)$ is an $r_{x}$-crossing, o(i) is an $r_{y^{-}}$or $s_{x}-$ crossing. If (i) is an $r_{y}$-crossing, then $o(i)$ is an $r_{x}-$ or $s_{y^{-c r o s s i n g}}$.

PROOF. The edge in question is part of an overcrossing segment at (i) which belongs to the $x-$ (resp. $y-$ ) component. At $O(i)$, this overcrossing segment must terminate. Thus $o(i)$ is an $r_{y^{-}}$or $s_{x^{-}}\left(\operatorname{resp} . r_{x^{-}}\right.$or $\left.s_{y^{-}}\right)$ crossing. 
COROLIARY 4.5. If $b_{0} \neq 0$, the number of off-diagonal factors $b_{i 0}$ coming from $r_{x}$-rows equals the number of off-diagonal elements coming from $r_{y}$-rows.

COROLLARY 4.6. If $b_{0} \neq 0$, then there is a positive integer $p_{1}$ and nonnegative integers $p_{2}, p_{3}, p_{4}, p_{5}$ such that

$$
b_{0}=(x-1)^{p_{1}^{-1}}(y-1)^{p_{1}}(-x)^{p_{2}}(-y)^{p_{3}}(x-1)^{p_{4}}(y-1)^{p_{5}} .
$$

The corresponding term of $\operatorname{det} \overline{\mathrm{A}}^{\prime}$ is

$$
a_{0}=(x-1)^{p_{1}}(y-1)^{p_{1}-1}(-x)^{p_{2}}(-y)^{p_{3}}(x-1)^{p_{4}}(y-1)^{p_{5}} \text {. }
$$

PROOF. In $B^{\prime}$, there are $R_{x}-1 \quad r_{x}$-rows and $R_{x} r_{y}$-rows. Since the offdiagonal factors of $b_{0}$ must be paired between $r_{x^{-}}$and $r_{y}$-rows, there must be an imbalance of 1 in the diagonal elements. In $a_{0}$, this imbalance is reversed.

THEOREM 4.7. $\operatorname{det} B^{\prime}=(1-y) \Delta(x, y)$, up to units in $\mathbb{Z}\left[x, x^{-1}, y, y^{-1}\right]$. PROOF. By Lemma 4.I, $\operatorname{det} \bar{A}^{\prime}=\sum_{0 \varepsilon \Sigma}(-1)^{0} a_{0}=(1-x) \Delta(x, y)$, up to units. Let $a_{0}=(x-1) a_{0}^{\prime}$. By Corollary 4.6, $b_{0}=(y-1) a_{0}^{\prime}$. Thus $\operatorname{det} B^{\prime}=$ $=\sum_{0}(-1)^{\sigma_{b}} b_{0}=(1-y) \Delta(x, y)$, up to units.

THEOREM 4.8. The polynomials $(1-y) \Delta(x, y)$ and $(1-x) \Delta(x, y)$ are alternating. That is, $(1-y) \Delta(x, y)$ can be written as $\sum_{i, j} c_{i j} x^{i} y^{j}$ in such a way that $(-1)^{i+j} c_{i j} \geq 0$.

PROOF. The argument is identical to $[1,(2.13), \mathrm{p} .262]$. By Theorem 3.1, we can compute $\operatorname{det} B^{\prime}=(1-y) \Delta(x, y)$ by summing the products of the labels on maximal rooted trees in the graph of $P$. One of these products is positive if and only if it has an even number of edges labelled "-x" or "-y". All the results we have developed for $(1-y) \Delta(x, y)$ can be duplicated for $(1-x) \Delta(x, y)$ by making vertex $(1)$ an $r_{y}$-crossing. 
It would be interesting to know whether the polynomial $\Delta(x, y)$ must be alternating for alternating links. This does not follow by elementary algebra from our results. For example, the polynomial

$$
\begin{gathered}
3-2 x+2 x^{2} \\
-2 y-x y-2 x^{2} y \\
+2 y^{2}-2 x y^{2}+3 x^{2} y^{2}
\end{gathered}
$$

becomes alternating upon multiplication by $(1-x)$ or $(1-y)$. According to the Torres conditions [5, p.57], this polynomial could be the Alexander polynomial of a linking-number-one link each of whose components has Alexander polynomial $3-5 t+3 t^{2}$.

\section{REFERENCES}

1. Crowell, R. H. Genus of alternating link types, Annals of Math. 69 (1959) $258-275$.

2. Crowell, R. H. and R. H. Fox. Introduction to Knot Theory, Ginn \& Company, New York, 1963.

3. Moon, J. W. Counting Labelled Trees, Canadian Mathematical Monographs No. I, William Clowes and Sons, London, 1970.

4. Rolfsen, D. Knots and Links, Mathematical Lecture Series No. 7, Publish or Perish, Berkeley, 1976.

5. Torres, G. On the Alexander polynomial, Annals of Math. 57 (1953) 57-89. 


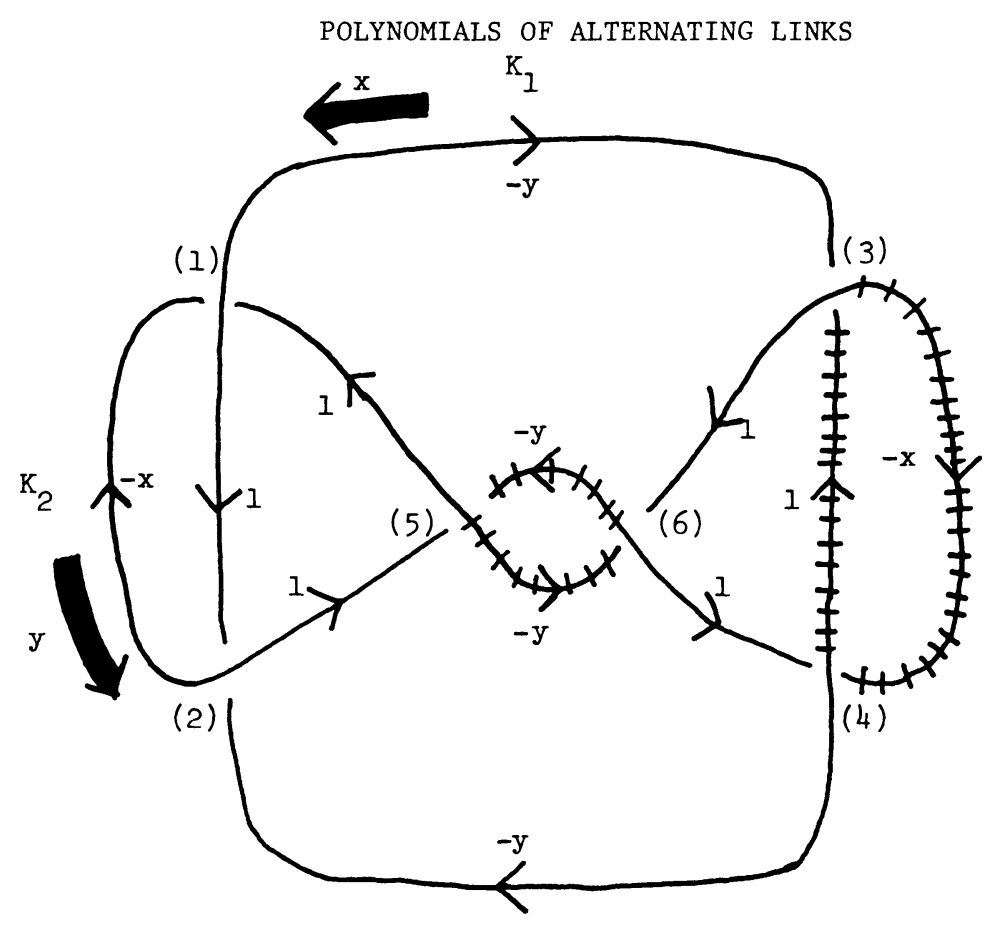

FIGURE 1

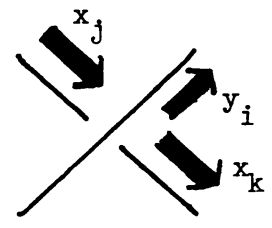

a) + crossing

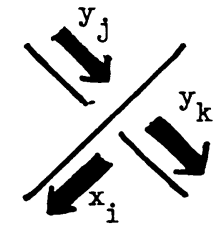

b) - crossing

FIGURE 2 


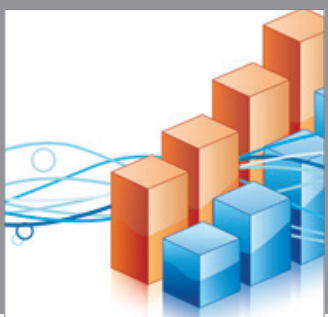

Advances in

Operations Research

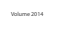

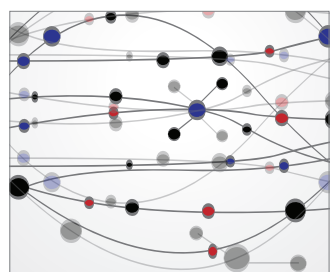

\section{The Scientific} World Journal
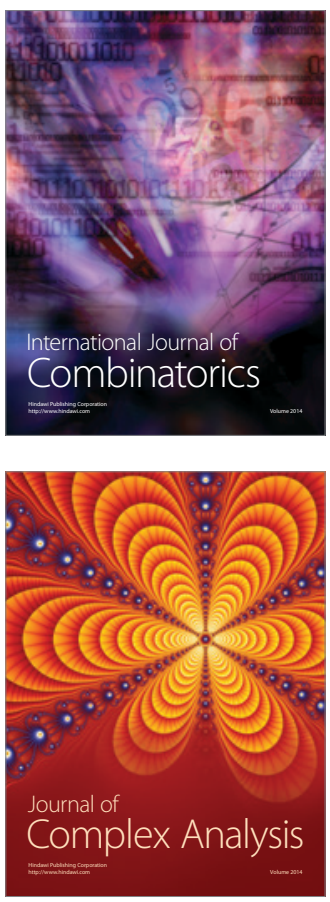

International Journal of

Mathematics and

Mathematical

Sciences
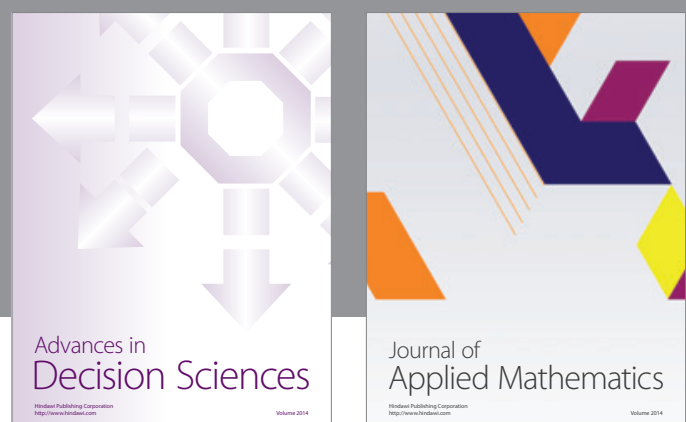

Journal of

Applied Mathematics
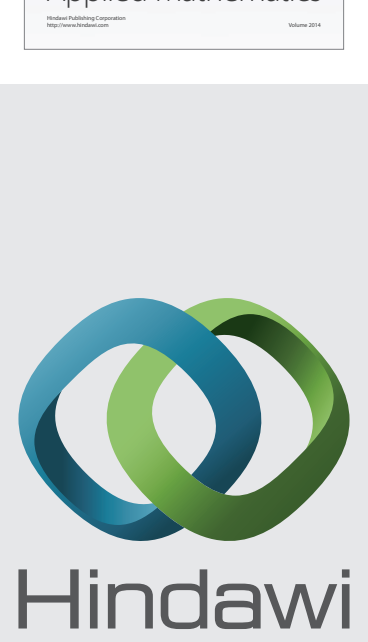

Submit your manuscripts at http://www.hindawi.com
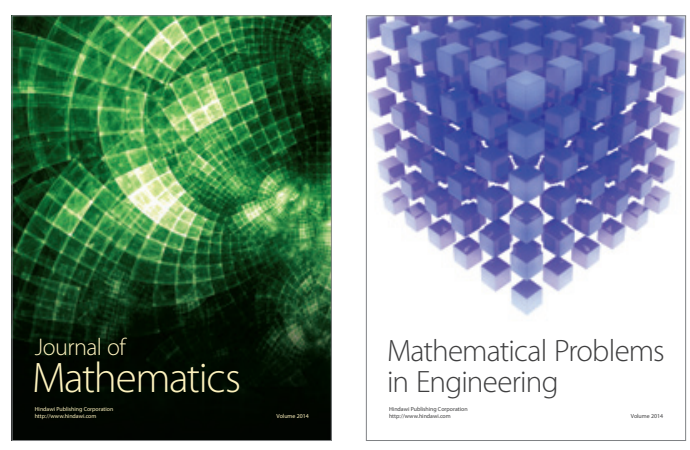

Mathematical Problems in Engineering
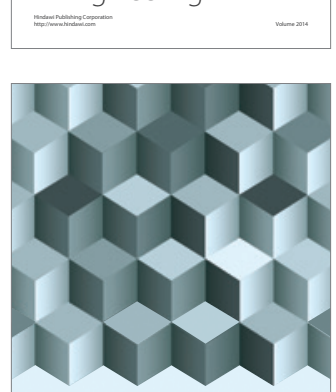

Journal of

Function Spaces
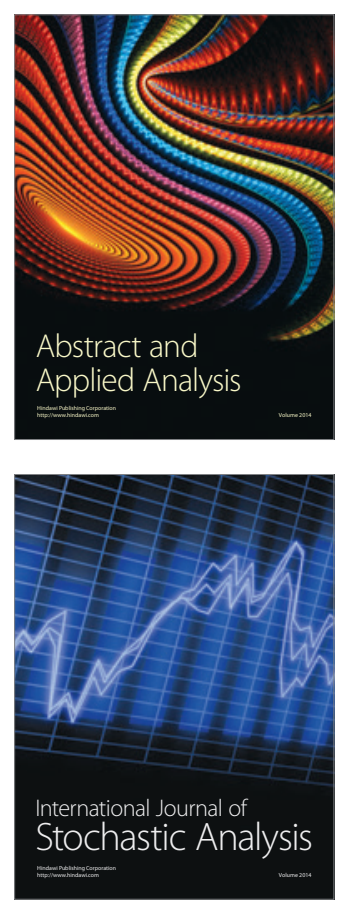

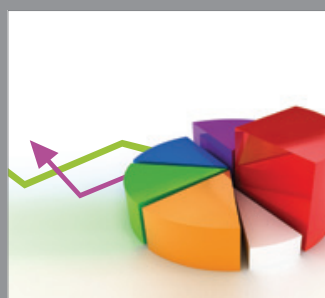

ournal of

Probability and Statistics

Promensencen
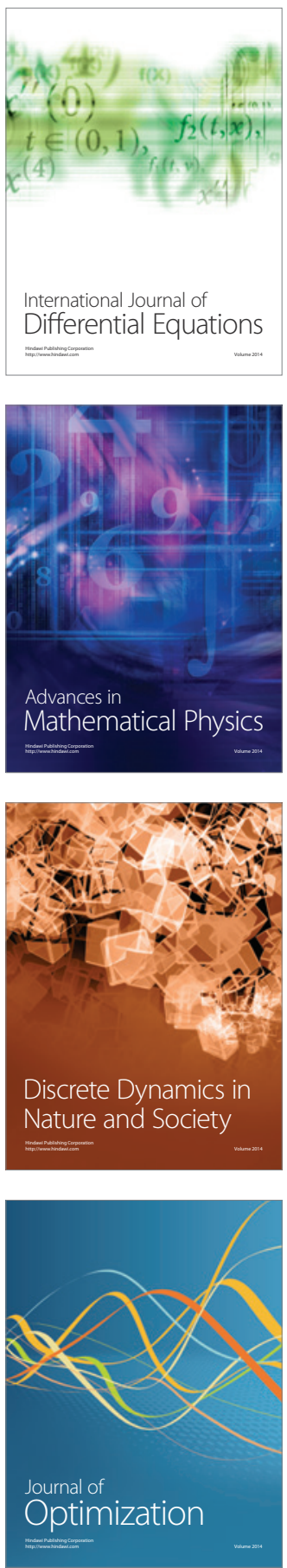